\title{
Attenuation of Glomerular Endothelial Cells from High Glucose-Induced Injury by Blockade of MAD2B
}

\author{
Yu-Mei Wang ${ }^{\mathrm{a}}$ Yu Hao ${ }^{\mathrm{a}}$ Xian-Fang Meng ${ }^{\mathrm{b}}$ Fang-Fang He $\mathrm{He}^{\mathrm{a}}$ Shan Chen \\ Pan Gao Hui Tang ${ }^{a}$ Hua Su ${ }^{a}$ Chun Zhang ${ }^{a}$
}

aDepartment of Nephrology, Union Hospital, Tongji Medical College, Huazhong University of Science and Technology, ${ }^{b}$ Department of Neurobiology, Tongji Medical College, Huazhong University of Science and Technology, Wuhan, China

\section{Key Words}

MAD2B $\cdot$ High glucose $\cdot$ Glomerular endothelial cells

\begin{abstract}
Background/Aims: To assess the role of mitotic arrest-deficient 2-like protein 2 (MAD2B) in high glucose-induced injury in mouse glomerular endothelial cells (GEnCs). Methods: GEnCs were cultured in vitro, and MAD2B protein levels were measured by Western blot in cells stimulated with high glucose $(30 \mathrm{mM})$ for various periods of time. MAD2B and scrambled shRNA were introduced into GEnCs by liposomal transfection. Cell proliferation, apoptosis, nitric oxide (NO) production, and monolayer permeability were then measured in cells grown in the following conditions: control, high glucose treatment, MAD2B shRNA transfection with high glucose treatment, and scrambled shRNA transfection with high glucose treatment. Results: High glucose increased the protein levels of MAD2B in GEnCs. Compared with control cells, apoptosis was increased by high glucose treatment, which was attenuated by transfection with $M A D 2 B$ shRNA transfection. Cells treated with high glucose produced less NO than control cells, whereas MAD2B shRNA transfection increased NO production. Cell monolayer permeability was enhanced in high glucose treated cells, but MAD2B shRNA transfection reduced permeability. Conclusion: High glucose levels induced the expression of MAD2B in GEnCs, whereas suppressing its expression reduced high glucose-induced endothelial cell apoptosis and high permeability, and promoted cell proliferation and NO production.
\end{abstract}


Wang et al.: MAD2B in Glomerular Endothelial Cells

\section{Introduction}

Diabetic nephropathy (DN) is one of the most common microvascular complications of diabetes mellitus, it has also become the second leading cause of end stage renal disease (ESRD) in China [1]. Due to the confounding metabolic syndrome, it is more difficult to treat diabetes-induced ESRD than other kidney diseases. Therefore, early prevention is important for delaying the development of DN.

The pathogenesis of DN is not fully understood. A large body of research suggests that hyperglycemia causes abnormalities in hemodynamics and glucose metabolism, which lead to kidney damage and pathogenic changes in the resident cells of the kidney glomeruli. The role of glomerular endothelial cells (GEnCs) in the development of DN has become the focus of many recent studies [2]. Because of their direct contact with the circulation, vascular endothelial cells directly receive and respond to metabolic, biochemical, and hemodynamic signals in the blood. A number of phenotypic changes have been reported in GEnCs under hyperglycemic conditions, including suppressed cell proliferation and increased apoptosis [3], altered endothelial nitric oxide synthase (eNOS) activity and nitric oxide (NO) production [4], enhanced cell monolayer permeability due to cytoskeletal changes [5], the secretion of cytokines such as angiotensin II (Ang II), transforming growth factor $\beta$ (TGF $\beta$ ), and interleukin 6 (IL-6) [6-8], and disruption of the cell surface glycocalyx [9]. Because endothelial cells are sensitive to blood glucose levels, hyperglycemia-induced endothelial injury and dysfunction form the initial step toward diabetic vascular complications. However, the exact molecular mechanisms underlying high glucose-induced GEnC dysfunction are not completely elucidated.

Previous data from our laboratory suggest that mitotic arrest-deficient 2-like protein 2 (MAD2B, also termed MAD2L2) is involved in the development of DN. MAD2B acquired its name due to its $23 \%$ sequence identity and $54 \%$ similarity to mitotic arrest-deficient 2 (MAD2) at the amino acid level [10]. Both of these proteins contain a HORMA structural domain, which is involved in protein-protein interactions, protein oligomerization, and chromatin state recognition. A yeast two-hybrid screen revealed that MAD2B interacts with CDH1 and CDC20, both of which are essential activators of the anaphase-promoting complex/cyclosome (APC/C). MAD2B regulates cell cycle progression by binding to CDH1 or CDC20 and inhibiting the activity of APC/C $[11,12]$. MAD2B levels are upregulated in many malignant tumors, and correlate with tumor stage and poor prognosis, probably due to mitotic failure and genetic instability caused by the inhibition of APC/C [13-15]. We previously used immunohistochemistry to demonstrate that MAD2B protein levels were increased in the kidney glomeruli of streptozotocin (STZ)-induced type 1 diabetic mice, the $\mathrm{db} / \mathrm{db}$ model of type 2 diabetes, and kidney biopsies of patients with diabetic nephropathy. In podocytes cultured in vitro, high glucose induced the expression of MAD2B, which mediated high glucose-induced injury by affecting the expression of APC/C substrates. In addition, the overexpression of MAD2B accentuated high glucose-induced podocyte injury and apoptosis, whereas inhibiting the expression of MAD2B attenuated such injury and apoptosis (paper in process). Despite the important role of endothelial cells in DN, the role of MAD2B in endothelial cells has not been studied. In the current study, we investigated the role of MAD2B in high glucose-induced GEnC injury, and discussed its potential association with DN.

\section{Materials and Methods}

Cell culture and treatment

Murine glomerular endothelial cells (GEnCs) were purchased from Cell Biologics Company (C576014G) and were cultured according to the manufacturer's protocol and previously reports [16]. Cells were grown in Dulbecco's modified Eagle's medium (DMEM) (Gibco Invitrogen, LifeTechnologies, Scotland, UK) supplemented with 10\% fetal bovine serum (FBS, Gibco Invitrogen). For high glucose treatment, cells were 
Wang et al.: MAD2B in Glomerular Endothelial Cells

dissociated with $0.25 \%$ trypsin-EDTA solution (Gibco Invitrogen) for $1-2$ min at $37^{\circ} \mathrm{C}$. As soon as the cells detached, cell culture media supplemented with $10 \%$ FBS were added, and the cells were plated in six-well plates coated with a gelatin-based coating solution in a humidified, $5 \% \mathrm{CO}_{2}$ incubator at $37^{\circ} \mathrm{C}$. Twelve hours later, cells were incubated in DMEM without FBS for another $24 \mathrm{~h}$. Cell were then incubated in $5.5 \mathrm{mM}$ D-glucose (control, normal glucose [NG] group), $\mathrm{NG}$ plus mannitol (5.5 mM glucose $+24.5 \mathrm{mM}$ mannitol; an osmotic control), or $30 \mathrm{mM}$ D-glucose (high glucose [HG] group) for 12, 24, or $48 \mathrm{~h}$. MAD2B levels were then determined by western blot as described below.

\section{Western blot}

Western blot was performed as described previously [17]. Briefly, protein concentrations were determined (BCA Protein Assay Kit, Beyotime, Shanghai), and $70 \mu \mathrm{g}$ protein samples were separated on $12 \%$ SDS-PAGE gels and transferred to polyvinylidene difluoride membranes (Sigma-Aldrich, St. Louis, MO, USA). Non-specific binding was blocked by incubating membranes in 5\% fat-free milk in TBS buffer containing $0.1 \%$ Tween 20 (TBS-T) for $1 \mathrm{~h}$. Membranes were then incubated overnight at $4^{\circ} \mathrm{C}$ with primary antibodies against MAD2B (1:1000, Rockland Immunochemicals Inc. Gibertsvile, PA, USA) and $\beta$-actin (1:4000, Santa Cruz Biotechnology, CA, USA). After washing, the blots were incubated for $1 \mathrm{~h}$ at room temperature (RT) with horseradish peroxidase (HRP)-labeled secondary antibodies (1:4000). Immunoreactive bands were developed using an enhanced chemiluminescence (ECL) Western blot detection reagent (Thermo Scientific, MA, USA). $\beta$-actin was used as the loading control. The density of each band was quantified using Image J software (NIH, Bethesda, MD, USA).

\section{Transient transfection of recombinant plasmids into GEnCs using liposomes}

GEnCs were plated into six-well plates $24 \mathrm{~h}$ before transfection, and were transfected when they became $80 \%$ confluent. Thirty minutes before transfection, the culture media were changed to OPTI-MEM (Gibco, USA). For transfection, $4 \mu \mathrm{g}$ of plasmid DNA (MAD2B shRNA or scrambled shRNA) was added to 50 $\mu \mathrm{l}$ OPTI-MEM. In a separate tube, $8 \mu \mathrm{l}$ of Lipofectamine $2000^{\text {TM }}$ (lipo 2000, Invitrogen, CA, USA) was added to $50 \mu \mathrm{l}$ of OPTI-MEM; both tubes were mixed and left to stand at RT for $5 \mathrm{~min}$. The diluted DNA and lipo 2000 solutions were then combined, gently mixed, and left to stand at RT for $20 \mathrm{~min}$. The mixture was then added into 6-well plates, which were gently shaken and then placed back into the incubator. Six hours after transfection, the media were replaced with complete media.

\section{Cell proliferation assay}

GEnCs were transfected in six-well plates as described above, and were dissociated into single-cell suspensions $6 \mathrm{~h}$ after transfection. The cells were re-plated into 96-well plates $(100 \mu \mathrm{l} /$ well, $\sim 5,000$ cells/ well) and incubated for $12 \mathrm{~h}$. The media were then replaced with $100 \mu \mathrm{l} /$ well fresh medium, and the cells were divided into four treatment groups: control, high glucose alone, high glucose plus scrambled shRNA transfection, and high glucose plus MAD2B shRNA transfection. For GEnCs proliferation assay, a colorimetric BrdU Cell Proliferation ELISA Kit (Abcam, Cambridge, UK) was used as described previously [18]. After $48 \mathrm{~h}$ treatment of high glucose, BrdU was added 24 hours before the end of the incubation period. After that, cells were fixed and BrdU content was assessed using a monoclonal anti-BrdU antibody according to the manufacturer's instructions. The absorbance at $450 \mathrm{~nm}$ was then measured using a microplate reader (TECAN, Männedorf, Switzerland).

\section{Apoptosis analysis using flow cytometry}

GEnCs were transfected in six-well plates as described above, and were then divided in to four treatment groups $6 \mathrm{~h}$ after transfection: control, high glucose alone, high glucose plus scrambled shRNA transfection, and high glucose plus MAD2B shRNA transfection. Cells were collected for analysis after 48 $\mathrm{h}$ of high glucose treatment. The culture media containing floating cells were removed from the wells and transferred into labeled tubes. The attached cells were then dissociated with $0.25 \%$ trypsin (no EDTA) and transferred into the same labeled tubes. Cells were centrifuged at 2,000 rpm for $5 \mathrm{~min}$ at RT, the supernatant was discarded and the cells were washed twice with PBS. The cells were centrifuged again, and resuspended in $500 \mu \mathrm{l}$ of binding buffer. To stain apoptotic cells, $1 \mu \mathrm{l}$ of annexin V-APC was added, followed by $5 \mu \mathrm{l}$ of 7-AAD buffer (Nanjing KeyGEN Biotech, China). Cells were stained in the dark for $15 \mathrm{~min}$, and then analyzed by flow cytometry (BD, Biosciences, CA, USA) within 1 hour.

\section{KARGER}


Quantification of NO levels in culture media (nitrate reductase assay)

GEnCs were transfected in six-well plates as described above, and were divided into four treatment groups: control, high glucose alone, high glucose plus scrambled shRNA transfection, and high glucose plus MAD2B shRNA transfection. The culture media were collected after 24 or $48 \mathrm{~h}$ of high glucose treatment, and the levels of NO in the media were measured using a NO quantification kit (Nanjing Jiancheng Biotech, China) following the manufacturer's instructions.

Cell monolayer permeability assay

GEnCs were transfected in six-well plates as described above, dissociated into single cell suspensions of $2 \times 10^{6}$ cells $/ \mathrm{ml}$ after $6 \mathrm{~h}$, and then plated into the upper chamber of Transwell plates in which the membranes (polycarbonate, pore size $0.4 \mu \mathrm{m}, 12 \mathrm{~mm}$ diameter) were coated with the matrix from an in vitro vascular permeability assay kit (Millipore, Bedford, MA, USA). Cells were divided into four treatment groups: control, high glucose alone, high glucose plus scrambled shRNA transfection, and high glucose plus $M A D 2 B$ shRNA transfection. A total of $500 \mu \mathrm{l}$ of the appropriate media were added to the lower chambers. When cells reached confluency $48 \mathrm{~h}$ later, the media were removed from both chambers without disturbing the cell monolayer; $150 \mu \mathrm{l}$ of FITC-dextran $(1 \mathrm{mg} / \mathrm{ml})$ was added to the upper chamber, and $500 \mu \mathrm{l}$ culture medium (without phenol red) was added to the lower chamber. The plates were left to stand at RT for 5 min, and then $100 \mu \mathrm{l}$ of the medium was removed from the lower chamber to measure the fluorescence intensity using a fluorescence spectrometer (Shanghai Jingke, China). The relative permeability of cells in each treatment group was calculated as the ratio of the mean intensity of the experimental group to the mean intensity of the control group.

Statistical analysis

All data are presented as means \pm standard deviations $(M \pm S D)$, and GraphPad Prism 5 was used for all statistical analyses. One-way analysis of variance (ANOVA) was used to compare differences among groups, and $p<0.05$ designated to indicate statistical significance.

\section{Results}

High glucose upregulates MAD2B in cultured GEnCs

Murine GEnCs were incubated in high glucose (30 mM) for $0,12,24$, or $48 \mathrm{~h}$, and MAD2B protein levels were measured by Western blot. MAD2B levels were significantly higher at all time points (12-48 h) following high glucose stimulation, and peaked at $24 \mathrm{~h}$ (Fig. 1A, B). However, MAD2B levels were unaffected by treatment with a high concentration of mannitol (24.5 mM mannitol + 5.5 mM glucose) (Fig. 1C, D), suggesting that the effect of high glucose on MAD2B expression was not simply due to high osmotic pressure.

\section{MAD2B shRNA effectively blocks MAD2B expression}

GEnCs were transfected with recombinant plasmids expressing MAD2B or scrambled shRNA, and Western blot was used to assess transfection efficiency after $48 \mathrm{~h}$. Compared with untransfected control cells, MAD2B protein levels were significantly lower in those transfected with $M A D 2 B$ shRNA, whereas expression was unchanged in those transfected with scrambled shRNA (Fig. 2).

MAD2B blockade promotes proliferation of high glucose-treated GEnCs

GEnCs were treated as described above, and incubated for $48 \mathrm{~h}$; cell proliferation was then determined using a BrdU Cell Proliferation ELISA Kit. The proliferation rate of high glucose-treated cells was declined significantly at $48 \mathrm{~h}$ comparable to that of control cells (Fig. 3). MAD2B shRNA-transfected cells proliferated faster under a high glucose environment than scrambled shRNA transfected cells, but remained slower than control cells (Fig. 3).

MAD2B blockade reduces apoptosis of high glucose-treated GEnCs

GEnCs were treated as described above, and apoptosis was analyzed using flow cytometry after $48 \mathrm{~h}$. Fig. 4A shows representative dot plots from each group. The lower 


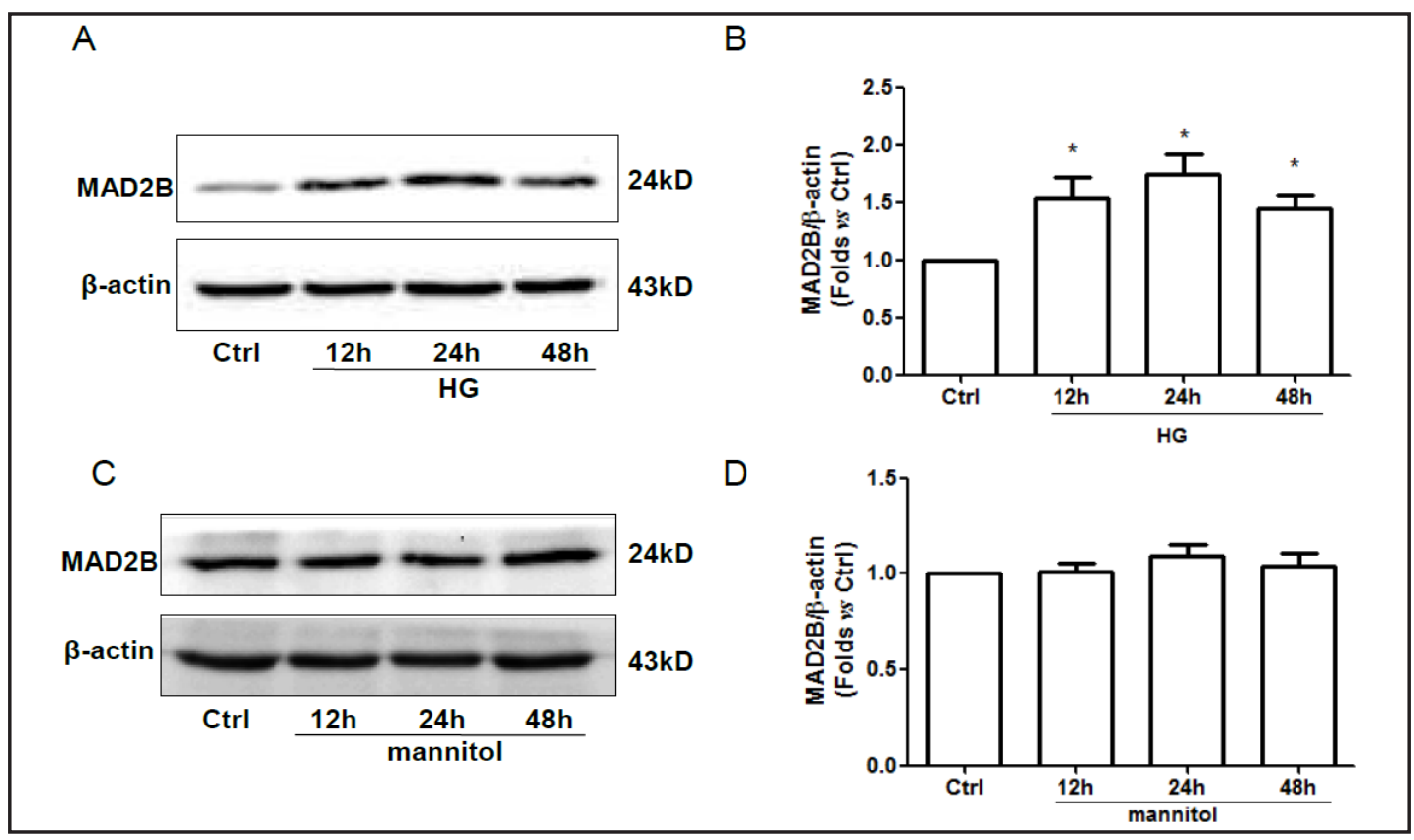

Fig. 1. High glucose increases MAD2B protein levels in glomerular endothelial cells (GEnCs). GEnCs were stimulated with high glucose (HG) (A-B) or high mannitol (C-D) for 0 (Ctrl), 12, 24, or $48 \mathrm{~h}$, and MAD2B expression was analyzed by Western blot. A, C, Representative images of western blots are shown. B, D, Bar graphs showing the results of statistical analysis. ${ }^{*} p<0.05$ vs. $\mathrm{Ctrl}(n=4$ in B, and $n=3$ in D).

Fig. 2. $M A D 2 B$ shRNA transfection decreases MAD2B protein levels in GEnCs. GEnCs were left untransfected (Ctrl), or were transfected with scrambled shRNA (Scra) or MAD2B shRNA (shRNA); MAD2B levels were

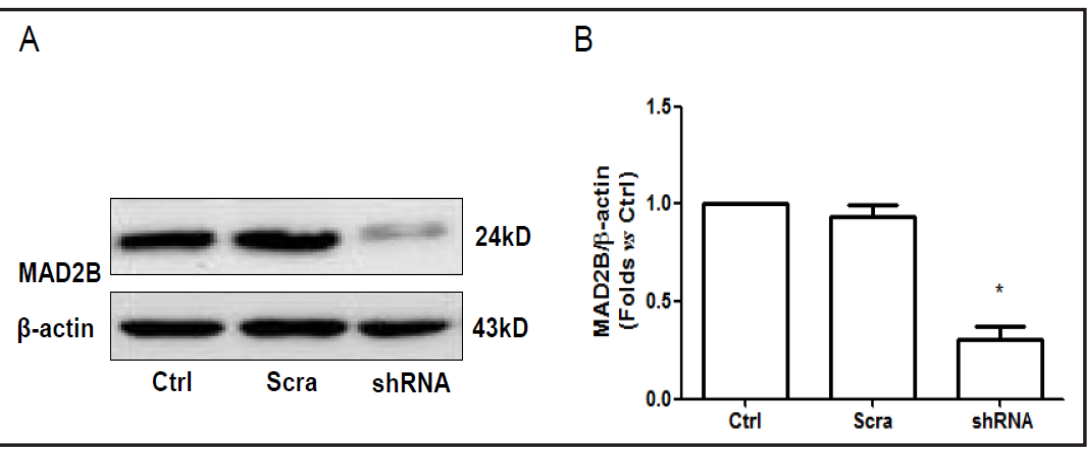
then analyzed by Western blot. A, Representative Western blot images. B, Bar graph showing the results of statistical analysis. $* p<0.05$ vs. Ctrl $(n=3)$.

Fig. 3. Knockdown of $M A D 2 B$ induces GEnC proliferation. GEnCs were grown in normal glucose (Ctrl), high glucose (HG), and/or transfected with scrambled shRNA (scra) or MAD2B shRNA (shRNA). Cell proliferation was then assessed after $48 \mathrm{~h}$ of high glucose treatment. ${ }^{*} p<0.05$ vs. Ctrl; $\# p<0.05$ vs. $\mathrm{HG}+$ scra at the same time point $(n=4)$.

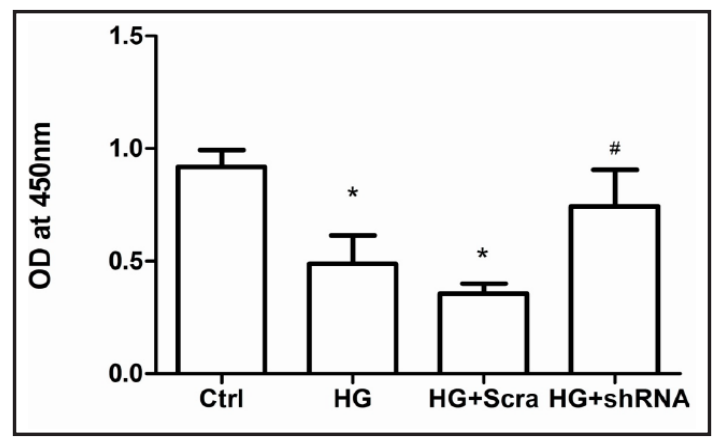

left quadrant contains live cells, the upper left quadrant includes cells that were damaged mechanically, the lower right quadrant represents early apoptotic cells, and the upper right quadrant contains late apoptotic and necrotic cells. The apoptotic index was calculated as the sum of the percentages of cells in the lower and upper right quadrants. Compared with 


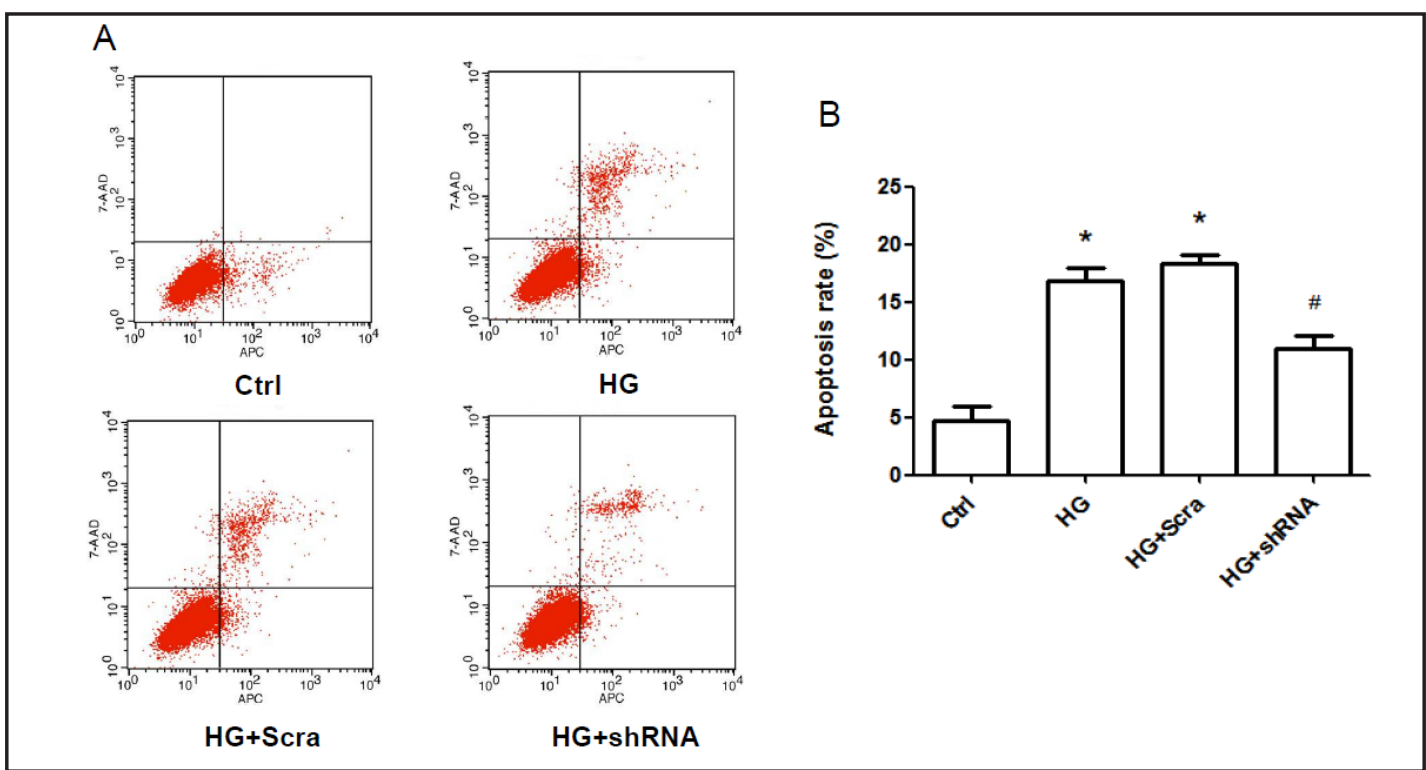

Fig. 4. Knockdown of MAD2B reduces apoptosis in GEnCs. GEnCs were grown in normal glucose (Ctrl), high glucose (HG), and/or transfected with scrambled shRNA (scra) or MAD2B shRNA (shRNA), and apoptosis was analyzed using flow cytometry. A, Representative dot plots showing annexin V-APC on the x-axis and 7 -AAD on the $\mathrm{y}$-axis. B, Bar graph showing the results of statistical analysis. ${ }^{*} p<0.05$ vs. Ctrl; \# $p<0.05$ vs. $\mathrm{HG}+\operatorname{scra}(n=3)$.

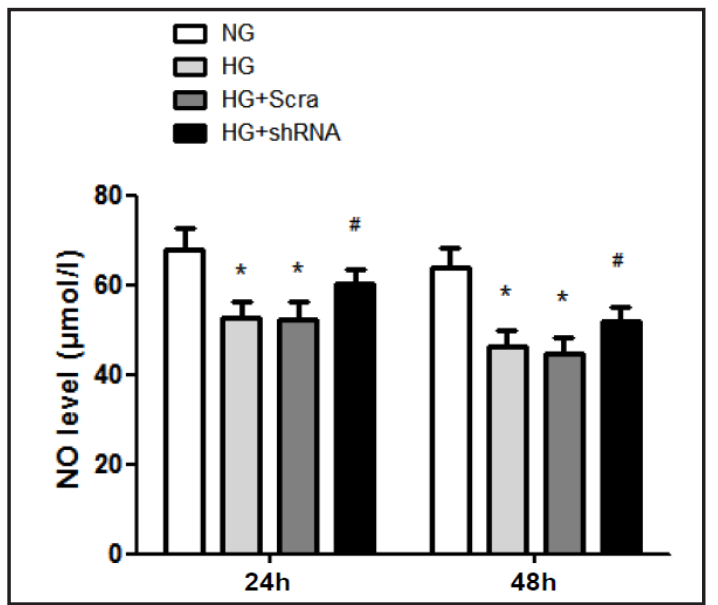

Fig. 5. Knockdown of $M A D 2 B$ induces nitric oxide (NO) production in GEnCs. GEnCs were grown in normal glucose (Ctrl), high glucose (HG), and/or transfected with scrambled shRNA (scra) or MAD2B shRNA (shRNA). The levels of NO secreted into the culture media were then analyzed 24 or $48 \mathrm{~h}$ after high glucose treatment. $* p<0.05$ vs. Ctrl; $\# p<0.05$ vs. $H G+$ scra at the same time point $(n=6)$.

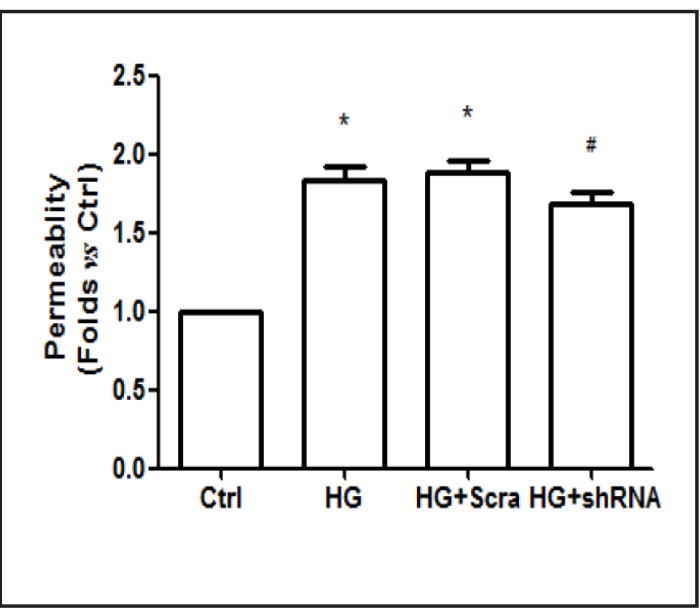

Fig. 6. Knockdown of $M A D 2 B$ reduces the monolayer permeability of GEnCs. GEnCs were grown in normal glucose (Ctrl), high glucose (HG), and/or transfected with scrambled shRNA (scra) or MAD2B shRNA (shRNA), and cell monolayer permeability was analyzed $48 \mathrm{~h}$ after high glucose treatment. $* p<0.05$ vs. Ctrl; $\# p<0.05$ vs. HG $+\operatorname{scra}(n=3)$.

control cells, the apoptotic index was significantly higher in cells treated with high glucose, whereas MAD2B shRNA-transfected cells had a lower apoptotic index than scrambled shRNA-transfected cells under the same high glucose conditions (Fig. 4B). This suggests that high glucose induces apoptosis in GEnCs, but that silencing MAD2B inhibits this effect. 
Wang et al.: MAD2B in Glomerular Endothelial Cells

MAD2B blockade induces NO production in high glucose-treated GEnCs

A nitrate reductase assay was used to measure the amount of NO secreted into the culture medium by cells in treatment groups described above. Measurements were taken after 24 or $48 \mathrm{~h}$ of high glucose treatment. Compared with control cells, the NO producing ability declined in GEnCs after high glucose treatment at both time points, whereas cells transfected with MAD2B shRNA secreted more NO than scrambled shRNA transfected cells under the same high glucose conditions (Fig. 5).

MAD2B blockade reduces monolayer permeability of high glucose-treated GEnCs

FITC-dextran was used as a tracking reagent in confluent GEnCs to assess cell monolayer permeability (Fig. 6). High glucose treatment significantly increased GEnCs monolayer permeability, whereas MAD2B shRNA transfection partially reversed this effect. This suggests that inhibiting MAD2B expression using shRNA could alleviate the increased endothelial permeability induced by high glucose.

\section{Discussion}

MAD2B is a member of the mitotic arrest deficient (MAD) family [10]. It interacts with many cellular proteins via its HORMA structural domain to regulate multiple cellular functions. Recently, a yeast two-hybrid screen revealed that MAD2B interacts with $\mathrm{CDH} 1$ and CDC20 [11], which both activate the APC/C complex. APC/C is an E3 ubiquitin ligase that plays a key role in the degradation of its target proteins via the ubiquitin-proteasome pathway. It regulates cell cycle progression by targeting cell cycle proteins, such as cyclin $\mathrm{B} 1$, cyclin A, p21, and p27, for polyubiquitination and subsequent proteasomal degradation [19]. MAD2B can inhibit APC/C activity by binding to CDH1 and CDC20; therefore, it is also involved in cell cycle control. In addition, MAD2B interacts with TCF4 and regulates the Wnt/ $\beta$-catenin signaling pathway [20]. Most studies on MAD2B have focused on its role in cancer and neurological diseases [13-15, 21-23], but it is still unknown about its expression and function in the kidney.

Because of their unique structure and function, the role of glomerular endothelial cells in kidney diseases has attracted significant attention. In many ways, DN may be viewed as a disease of the endothelial cell [24]. During DN, hyperglycemia and products of abnormal glucose metabolism activate a number of signaling molecules, transcription factors, and inflammatory mediators that together form a complex signaling network that induces glomerular endothelial dysfunction and injury [25]. The major changes in endothelial cell structure and function under high glucose that lead to progressive fibrosis, reduced capillary perfusion, and diminished GFR [26]. In the current study, we demonstrated that MAD2B levels were upregulated in GEnCs by high glucose, peaking at $24 \mathrm{~h}$, and that this effect was not caused by high osmotic pressure.

Over the last few years, many in vitro studies focusing mainly on human or animal endothelial cells of kidney, retina, myocardium, and human umbilical vein endothelial cells (HUVECs) showed that hyperglycemia can induce endothelial cell apoptosis [27-29]. These mechanisms of apoptosis include oxidative stress, increased intracellular $\mathrm{Ca}^{2+}$, mitochondrial dysfunction, changes in intracellular fatty acid metabolism, activation of mitogen activated protein kinases (MAPK) signaling pathways, and impaired phosphorylation activation of the protein kinase Akt [30-32]. In our study, we found hyperglycemia induced GEnCs apoptosis, in addition, we also observed that MAD2B shRNA reduced high glucose-induced apoptosis. Previous studies revealed that high glucose could induce the expression of MAD2B in podocytes and neurons, and that MAD2B mediates high glucose-induced apoptosis [22, 23]. Mechanistically, the binding of MAD2B to CDH1 inhibits APC/C activity, leading to the abnormal accumulation of the APC/C substrate cyclin B1. The cyclin B1-CDK1 complex can promote the phosphorylation of the apoptotic protein BAD at serine 128 , which in 
turn induces apoptosis $[22,23]$. It is possible that MAD2B mediates high glucose-induced apoptosis in GEnCs via a similar mechanism.

In vitro, high glucose has been demonstrated to delay endothelial cell replication [33]. Culturing both human aortic endothelial cells (HAECs) and human umbilical vein endothelial cells (HUVECs) cells in high glucose increases the production of superoxide anion and also inhibits cell proliferation [34]. The results of our cell proliferation assay revealed that treatment with high glucose for $48 \mathrm{~h}$ inhibited GEnC proliferation, but that depleting MAD2B with shRNA promoted proliferation. In other tissues, studies assessing on the role of MAD2B in cell proliferation yielded conflicting results. In ovarian clear cell carcinoma cells, depleting MAD2B using shRNA reduced cell proliferation [21]. However MAD2B gene knockdown had no effect on the growth of nasopharyngeal carcinoma cells [15]. In intestinal epithelial cells infected with Shigella, MAD2B binds to IpaB, an effector protein secreted by Shigella, which releases MAD2B from CDH1 and CDC20 and leads to the over-activation of APC/C by CDH1 and CDC20. Therefore, Shigella disrupts the timely activation of APC/C and normal mitosis, causing intestinal epithelial cells to lose their ability for rapid self-renewal, which is their innate defense mechanism for controlling bacterial infection [35]. These results suggest that the role of MAD2B in cell proliferation is cell type-specific, probably because MAD2B binds to and regulate proteins from different signaling pathways in different cells.

In addition to partially reversing the effect of high glucose on cell proliferation and apoptosis, depleting MAD2B in GEnCs using shRNA also reversed the effect of high glucose on NO synthesis and monolayer permeability via unknown mechanisms. NO is a short-lived gaseous lipophilic molecule and is a paracrine mediator formed from its precursor L-arginine, which includes three NOS isoforms: neuronal (nNOS), inducible (iNOS) and endothelial (eNOS) [36]. NO is an important endothelium-derived mediator and with multiple actions, such as, vasodilation and antiproliferative, permeability-decreasing, and anti-inflammatory properties [37, 38]. Previous studies have provided conflicting results for the NO expression in diabetes. Most reports suggested that NO production is increased in early diabetes but decreased in advanced nephropathy [36]. Both eNOS and nNOS can contribute to the altered NO production, particularly the first [36]. Our study showed that HG decreased NO production, which is consistent with previous reports by Cheng and Hoshiyama et al [4, 38]. Decreased NO production and bioavailability might be associated with overproduction of superoxide and L-arginine deficiency, increased in microvessel permeability, resulting in impaired endothelial function [4, 36], and which, may contribute to DN progression [37, 39]. MAD2B can interact with many proteins through its HORMA domain, executing a diverse array of biological functions. Some of the signaling pathways regulated by MAD2B might affect eNOS activity and cytoskeletal structure, which in turn regulate NO production and endothelial permeability, respectively.

In summary, this study demonstrated that high glucose induced MAD2B expression in GEnCs, and that depleting MAD2B using shRNA could alleviate high glucose-induced endothelial injury, including effect on cell proliferation, apoptosis, NO production, and monolayer permeability. These data suggest that MAD2B mediates high glucose-induced GEnC injury. This is the first report describing a role for MAD2B in kidney glomerular endothelial cells; however its mechanism of action awaits further investigation.

\section{Acknowledgement}

This work was supported by Grants from the National Natural Science Foundation of China (No. 30871174, No. 30800523, No. 81170662, No. 81170600, No. 31200872, No. 81300604, No. 81470964 and No. 81400720), the Natural Science Foundation of Hubei Province (No. 2013 CFA026 and No. 2012 FFA038), and a Doctoral Fund of Ministry of Education of China (No. 20130142110064). 


\section{Cellular Physiology and Biochemistry}

\section{Disclosure Statement}

All of the authors declared that there was no conflict of interest.

\section{Reference}

$\rightarrow 1$ Rossing P: Diabetic nephropathy: Worldwide epidemic and effects of current treatment on natural history. Curr Diab Rep 2006;6:479-483.

2 Eleftheriadis T, Antoniadi G, Pissas G, Liakopoulos V, Stefanidis I: The renal endothelium in diabetic nephropathy. Ren Fail 2013;35:592-599.

-3 Isermann B, Vinnikov IA, Madhusudhan T, Herzog S, Kashif M, Blautzik J, Corat MA, Zeier M, Blessing E, Oh J, Gerlitz B, Berg DT, Grinnell BW, Chavakis T, Esmon CT, Weiler H, Bierhaus A, Nawroth PP: Activated protein c protects against diabetic nephropathy by inhibiting endothelial and podocyte apoptosis. Nat Med 2007;13:1349-1358.

4 Hoshiyama M, Li B, Yao J, Harada T, Morioka T, Oite T: Effect of high glucose on nitric oxide production and endothelial nitric oxide synthase protein expression in human glomerular endothelial cells. Nephron Exp Nephrol 2003;95:e62-68.

5 Nitta K, Horiba N, Uchida K, Horita S, Hayashi T, Kawashima A, Yumura W, Nihei H: High glucose modulates albumin permeability across glomerular endothelial cells via a protein kinase c-dependent mechanism. Nihon Jinzo Gakkai Shi 1995;37:317-322.

6 Peng H, Xing YF, Ye ZC, Li CM, Luo PL, Li M, Lou TQ: High glucose induces activation of the local reninangiotensin system in glomerular endothelial cells. Mol Med Rep 2014;9:450-456.

7 Qiu HY, Fan WX, Zuo C, Huang SM, Liu F, Tang WX: [influence of mannose binding lectin to expression of tgfbeta1 and nf-kappab in high concentration of glucose cultured human renal glomerular endothelial cells]. Sichuan Da Xue Xue Bao Yi Xue Ban 2011;42:604-609.

-8 Wu XH, Huang SM, Fan WX, Tang WX, Qiu HY: [influence of high glucose and mannose binding lectin complement pathway activation to il- 6 and tnf-alpha's expression by human renal glomerular endothelial cells]. Sichuan Da Xue Xue Bao Yi Xue Ban 2011;42:90-94.

-9 Qiu HY, Fan WR, Huang SM, Liu F, Tang WZ, Zuo C: [effect of high concentration of glucose on thickness of glycocalyx and expression of syndecan-1 and glypican-1 in cultured human renal glomerular endothelial cells]. Sichuan Da Xue Xue Bao Yi Xue Ban 2010;41:980-985.

10 Cahill DP, da Costa LT, Carson-Walter EB, Kinzler KW, Vogelstein B, Lengauer C: Characterization of mad2b and other mitotic spindle checkpoint genes. Genomics 1999;58:181-187.

11 Chen J, Fang G: Mad2b is an inhibitor of the anaphase-promoting complex. Genes Dev 2001;15:1765-1770.

12 Pfleger CM, Salic A, Lee E, Kirschner MW: Inhibition of cdh1-apc by the mad2-related protein mad212: A novel mechanism for regulating cdh1. Genes Dev 2001;15:1759-1764.

13 Zhao J, Liu S, Wang H, Zhang X, Kang T, Li Z, Deng H, Yue W, Cao S: Mitotic arrest deficient protein mad2b is overexpressed in human glioma, with depletion enhancing sensitivity to ionizing radiation. J Clin Neurosci 2011;18:827-833.

14 Rimkus C, Friederichs J, Rosenberg R, Holzmann B, Siewert JR, Janssen KP: Expression of the mitotic checkpoint gene mad2l2 has prognostic significance in colon cancer. Int J Cancer 2007;120:207-211.

$>15$ Cheung HW, Chun AC, Wang Q, Deng W, Hu L, Guan XY, Nicholls JM, Ling MT, Chuan Wong Y, Tsao SW, Jin DY, Wang X: Inactivation of human mad2b in nasopharyngeal carcinoma cells leads to chemosensitization to DNA-damaging agents. Cancer Res 2006;66:4357-4367.

16 Luo C, Li T, Zhang C, Chen Q, Li Z, Liu J, Wang Y: Therapeutic effect of alprostadil in diabetic nephropathy: Possible roles of angiopoietin-2 and il-18. Cell Physiol Biochem 2014;34:916-928.

17 Wang Y, Landheer S, van Gilst WH, van Amerongen A, Hammes HP, Henning RH, Deelman LE, Buikema H: Attenuation of renovascular damage in zucker diabetic fatty rat by nwt-03, an egg protein hydrolysate with ace- and dpp4-inhibitory activity. PLoS One 2012;7:e46781.

18 Mesti T, Savarin P, Triba MN, Le Moyec L, Ocvirk J, Banissi C, Carpentier AF: Metabolic impact of antiangiogenic agents on u87 glioma cells. PLoS One 2014;9:e99198. 
Wang et al.: MAD2B in Glomerular Endothelial Cells

19 Barford D: Structure, function and mechanism of the anaphase promoting complex (apc/c). Q Rev Biophys 2011;44:153-190.

20 Hong CF, Chou YT, Lin YS, Wu CW: Mad2b, a novel tcf4-binding protein, modulates tcf4-mediated epithelialmesenchymal transdifferentiation. J Biol Chem 2009;284:19613-19622.

-21 Niimi K, Murakumo Y, Watanabe N, Kato T, Mii S, Enomoto A, Asai M, Asai N, Yamamoto E, Kajiyama H, Shibata K, Kikkawa F, Takahashi M: Suppression of rev7 enhances cisplatin sensitivity in ovarian clear cell carcinoma cells. Cancer Sci 2014;105:545-552.

22 Meng X, Tian X, Wang X, Gao P, Zhang C: A novel binding protein of single-minded 2: The mitotic arrestdeficient protein mad2b. Neurogenetics 2012;13:251-260.

-23 Meng X, Wang X, Tian X, Yang Z, Li M, Zhang C: Protection of neurons from high glucose-induced injury by deletion of mad2b. J Cell Mol Med 2014;18:844-851.

24 Gilbert RE: The endothelium in diabetic nephropathy. Curr Atheroscler Rep 2014;16:410.

25 Brownlee M: The pathobiology of diabetic complications: A unifying mechanism. Diabetes 2005;54:16151625.

26 Gilbert RE: Endothelial loss and repair in the vascular complications of diabetes: Pathogenetic mechanisms and therapeutic implications. Circ J 2013;77:849-856.

-27 Baumgartner-Parzer SM, Wagner L, Pettermann M, Grillari J, Gessl A, Waldhausl W: High-glucose--triggered apoptosis in cultured endothelial cells. Diabetes 1995;44:1323-1327.

28 Du XL, Sui GZ, Stockklauser-Farber K, Weiss J, Zink S, Schwippert B, Wu QX, Tschope D, Rosen P: Introduction of apoptosis by high proinsulin and glucose in cultured human umbilical vein endothelial cells is mediated by reactive oxygen species. Diabetologia 1998;41:249-256.

-29 Lorenzi M, Cagliero E, Toledo S: Glucose toxicity for human endothelial cells in culture. Delayed replication, disturbed cell cycle, and accelerated death. Diabetes 1985;34:621-627.

-30 Bucala R, Tracey KJ, Cerami A: Advanced glycosylation products quench nitric oxide and mediate defective endothelium-dependent vasodilatation in experimental diabetes. J Clin Invest 1991;87:432-438.

-31 Shin Shin Y, Baek SH, Chang KY, Park CW, Yang CW, Jin DC, Kim YS, Chang YS, Bang BK: Relations between enos glu298asp polymorphism and progression of diabetic nephropathy. Diabetes Res Clin Pract 2004;65:257-265.

-32 Nakagawa T, Tanabe K, Croker BP, Johnson RJ, Grant MB, Kosugi T, Li Q: Endothelial dysfunction as a potential contributor in diabetic nephropathy. Nat Rev Nephrol 2011;7:36-44.

-33 Lorenzi M, Nordberg JA, Toledo S: High glucose prolongs cell-cycle traversal of cultured human endothelial cells. Diabetes 1987;36:1261-1267.

-34 Zanetti M, Zwacka R, Engelhardt J, Katusic Z, O'Brien T: Superoxide anions and endothelial cell proliferation in normoglycemia and hyperglycemia. Arterioscler Thromb Vasc Biol 2001;21:195-200.

-35 Iwai H, Kim M, Yoshikawa Y, Ashida H, Ogawa M, Fujita Y, Muller D, Kirikae T, Jackson PK, Kotani S, Sasakawa C: A bacterial effector targets mad212, an apc inhibitor, to modulate host cell cycling. Cell 2007;130:611-623.

-36 Dellamea BS, Leitao CB, Friedman R, Canani LH: Nitric oxide system and diabetic nephropathy. Diabetol Metab Syndr 2014;6:17.

37 Stehouwer CD: Endothelial dysfunction in diabetic nephropathy: State of the art and potential significance for non-diabetic renal disease. Nephrol Dial Transplant 2004;19:778-781.

38 Cheng H, Wang H, Fan X, Paueksakon P, Harris RC: Improvement of endothelial nitric oxide synthase activity retards the progression of diabetic nephropathy in db/db mice. Kidney Int 2012;82:1176-1183.

-39 Baylis C: Nitric oxide deficiency in chronic kidney disease. Am J Physiol Renal Physiol 2008;294:F1-9. 